\title{
MARIE-VICTOIRE NANTET, Camille et Paul Claudel, Lignes de partage
}

\section{Catherine Mayaux}

\section{(2) OpenEdition}

\section{Journals}

\section{Édition électronique}

URL : https://journals.openedition.org/studifrancesi/45605

DOI : 10.4000/studifrancesi.45605

ISSN : 2427-5856

Éditeur

Rosenberg \& Sellier

\section{Édition imprimée}

Date de publication : 1 août 2021

Pagination : 420-421

ISSN : 0039-2944

\section{Référence électronique}

Catherine Mayaux, «MARIE-VIctolRe nANTET, Camille et Paul Claudel, Lignes de partage », Studi Francesi [En ligne], 194 (LXV | II) | 2021, mis en ligne le 01 septembre 2021, consulté le 15 octobre 2022. URL : http://journals.openedition.org/studifrancesi/45605; DOI : https://doi.org/10.4000/studifrancesi. 45605

Ce document a été généré automatiquement le 15 octobre 2022.

\section{(c)}

Creative Commons - Attribution - Pas d'Utilisation Commerciale - Pas de Modification 4.0 International - CC BY-NC-ND 4.0

https://creativecommons.org/licenses/by-nc-nd/4.0/ 


\title{
MARIE-VICTOIRE NANTET, Camille et Paul Claudel, Lignes de partage
}

\author{
Catherine Mayaux
}

\section{RÉFÉRENCE}

MARIE-VICTOIRE NANTET, Camille et Paul Claudel, Lignes de partage, Paris, Gallimard, 2020,

"Nouvelle Revue Française", 237 pp.

1 C'est un très beau livre que publie MARIE-VICTOIRE NANTET sous la couverture blanche filetée des éditions Gallimard, original dans sa forme et différent dans son propos de tout ce qu'on a pu lire à ce jour sur les deux génies issus de la même famille, Camille et Paul Claudel. Ni biographie, bien que l'auteur n'omette pas la dimension factuelle, ni dossier d'archives - correspondances, brouillons et documents y sont cependant scrutés -, ni réinvention narrative, même si la finesse de l'analyse ouvre aux interprétations. L'essai se concentre sur tout ce qui relie ces deux êtres exceptionnels, $\mathrm{du}$ tréfonds de leur filiation et de leur enfance communes, aux affres de la création dont ils ont tous deux affronté la puissance et les menaces. Le sous-titre Lignes de partage désigne tout ce qui a été partagé entre eux de l'enfance à l'adolescence, ou départagé au gré des engagements de leur jeunesse, autant que la ligne de partage des eaux qui a scindé leurs destinées: de ces deux jeunes gens pareillement dotés par leur milieu et leurs dons d'artistes, l'un a arpenté les chemins de la célébrité et s'est accompli, l'autre a glissé sur la pente de la folie et de la déperdition, sans que jamais se renoue le fil de l'intelligence profonde qui les unissait. Mais est-ce bien certain? Se sont-ils totalement sentis séparés l'un de l'autre pour autant? interroge ce livre: quels signes d'une communion enfouie mais continue frémissent au fil des années, des échanges, des souvenirs, et surtout des œuvres? C'est là que se situe la méditation de Marie-Victoire Nantet, armée de tout le savoir cumulé et décanté des études passées, et réfléchissant à profondeur d'intériorité sur les gestes, les mots, les figures, les écrits ou les cris qui jalonnent les deux vies et les deux œuvres examinées en miroir. C'est la réfraction de Camille sur Paul et de Paul sur Camille, de l'un au cœur de l'autre et dans 
son œuvre qui est ici exfiltrée des scories du temps et des projections de toutes sortes, et qui exhausse le sentiment commun de leur solitude fondamentale, décalage pour l'un, arrachement au monde pour l'autre. Les neuf chapitres suivent leur devenir croisé au fil de l'émergence des deux œuvres et de leur visibilité croissante dans le champ artistique et littéraire - jusqu'au moment tragique où la maladie de Camille contraint à son internement. Mais ce sont surtout les tessons d'une fouille archéologique qui refont surface sous la fine plume de l'essayiste et reconstituent peu à peu ce lien perdu, du moins ses traces. Jeune Romain est ainsi moins l'histoire de la sculpture que Camille fit de Paul à seize ans, pourtant remarquée lors de son exposition en 1886, que le mythe qui se construit rétroactivement autour de l'auteur de Tête d'or, dont le protagoniste se confond dans les esprits avec la figure sculptée comme avec son auteur - Marcel Schwob peint même son plâtre en or -: les deux génies créateurs seront louangés dans un même article d'Octave Mirbeau en 1893, n'était que Camille est désignée comme «l'élève de Rodin, et la sœur de M. Paul Claudel». L'accueil enthousiaste des hommes de lettres se renouvellera au moment de la parution de Partage de midi, sans que le lien soit fait avec un autre buste sculpté par Camille, Paul Claudel à 37 ans, pourtant reflet d'un Mesa déjà rincé par la vie. Mais au Brésil en 1918, si la photographie prise par Perrin de Paul, poitrine découverte, enthousiasme tant l'écrivain, c'est qu'elle avive, fût-ce inconsciemment, la mémoire des bustes de posture antique sculptés par sa sœur. De même, le souvenir de la somptueuse photographie de celle-ci dans sa jeunesse, prise par César, resurgit et bouleverse le vieil écrivain au milieu de ses confidences à Jean Amrouche publiées dans Mémoires improvisés. Cet entrecroisement des échos (res)suscités par les œuvres, des représentations de chacun et du jeu des ressemblances saisies de l'un à l'autre par l'accessoire d'un bicorne, d'un regard, d'un mot ou d'une posture est un fil rouge qui court au cœur de la relation de Camille et Paul. L'étude de leur sentiment d'autochtonie, que la perte et les malheurs rendent au fil du temps plus nostalgique, plonge au cœur d'un pays, d'une famille, d'ancêtres et de récits recueillis de la même bonne, qui façonnèrent leur éveil à la création et leurs manières d'être à leur entrée sur la scène parisienne. Chansons populaires de la servante, accent, intonation et parlure similaires, austérité des attaches vosgiennes comme du Tardenois auxquels tous deux se sentent intimement reliés, expliquent des débuts intriqués: Camille a d'abord portraituré au fusain quelques ancêtres paternels et ses parents, Paul immisce dans La Jeune fille Violaine et dans la trilogie des Coûfontaine des scènes de la vie familiale ou villageoise. La boiterie attribuée à Camille, comme pour mieux la rattacher à son patronyme, hante L'Otage autant que plus tard Le Soulier de satin (Prouhèze et Rodrigue à la fin), faisant planer l'ombre d'une sœur perdue dans l'intimité de l'œuvre. En 1943, quelques mois avant la mort de Camille, sa présence s'impose lors du projet de mise en scène de la pièce par Barrault: le dramaturge donne explicitement en référence, pour la scène de l'Ombre double, la figure féminine du Sakountala sculpté par sa sœur. Les amours réels et transfigurés de Paul et Rosalie, Ysé et Mesa, Prouhèze et Rodrigue, autant que de Camille et Rodin, «se rés[olvent] dans un geste ultime de femme», réminiscence de la figure «en laquelle Camille Claudel s'est incarnée, son frère l'a dit» (p. 186). Divers moments sont revisités au peigne fin comme le séjour à l'île de Wight en compagnie de Florence Jeans, et les textes et correspondances auxquels il donne lieu, le mariage de Louise en 1888 et le jeu double de Camille au sein de sa famille tandis que Paul est encore en quête de lui-même, l'exposition universelle de 1889 visitée ensemble et le lien croisé à Debussy, un étrange dîner dans l'atelier de Camille au retour d'Amérique de Paul en 1895, moment où tous 
deux commencent d'être connus. Le bilan dégage leur égale solitude, l'une écartée du monde par sa passion pour Rodin et sa paranoïa naissante, l'autre, par le fait Alpha, l'appel de Dieu entendu le jour de Noël 1886 et le désir d'exil qui le déportera sans cesse loin de France. D'une œuvre l'autre, les motifs circulent et les quelques lettres qui subsistent de leurs échanges témoignent de partages inspirants, quoiqu'à sens unique: la lettre de Camille à Paul de décembre 1893 «confie [s]es trouvailles» tout en se plaignant «que son frère ne lui parle jamais de ce qu'il écrit» (p. 81). Paul en revanche écrit dès 1905 sur l'œuvre de la statuaire, dans L'Occident, apportant par son génie intuitif un soutien fort à une sœur qui commence à s'épuiser. D'autres textes de Claudel semblent hantés par la présence de Camille et sa passion malheureuse pour Rodin, prémonitoire de la sienne propre pour Rosalie: le «Morceau d'un drame» réduit à quelques scènes elliptiques d'une pièce Une mort prématurée met en scène le trio d'un frère, d'une sœur et de son amant, en une allusion très incertaine au vécu. Dans le drame La Jeune fille Violaine (1892), si imprégné de l'atmosphère de Villeneuve, Violaine semble la figure inversée de Camille, plus proche peut-être par son caractère exigeant de sa sœur Bibiane, même si l'attribution des rôles est plus complexe qu'il n'y paraît: rejet et admiration, crainte et envie qui agitent le drame transposent indirectement les préoccupations d'un jeune créateur aux prises avec la personnalité altière et dominatrice de sa sœur dans un milieu familial impuissant. Le déplacement des positions des personnages dans la seconde version de La Jeune fille Violaine (1899), écrite sept ans plus tard, reflète l'infléchissement du jugement du jeune poète, et plus encore dans la triste comédie des adieux de la version renouvelée douze ans après dans L'Annonce faite à Marie (1911): du départ au rejet de Violaine, l'évolution semble annoncer par prémonition la mise au ban, puis la chute misérable de sa sœur qui surviendra en 1913. C'est aussi que l'angoisse de la folie hante l'écrivain qui se sent d'autant plus menacé qu'il voit sa sœur sombrer. Dès l'appel lancé par son père en 1904, l'appréhension des désordres mentaux de Camille en 1905, l'année même où lui-même traverse l'épreuve de l'abandon par Rosalie comme un ébranlement de sa raison, Paul se sent choqué, et le malaise persistera tout au long de sa vie, quoi qu'on en dise, et quelque représentation que le public en ait donné. Marie-Victoire Nantet, sans nier la difficulté de la position d'un diplomate qui, pour distant qu'il soit, aurait peut-être pu faire plus ou mieux, traque dans les écrits du poète et dramaturge, du critique pictural, et de l'exégète de la Bible, les témoignages de son anxiété, de ses hantises, de ses regrets et chagrins. La lecture de «Ma sœur Camille» écrit en 1951, mais aussi de passages de Seigneur, apprenez-nous à prier, du commentaire du Cantique des cantiques, de La Rose et le rosaire comme de son analyse lumineuse des œuvres sculptées de sa sœur nourrissent la réflexion et éloignent toute possibilité d'un jugement simplificateur. Sans éluder le poids des fantasmes qui pèsent sur une histoire gémellée, notamment dans l'échec amoureux, l'auteur invite à une «rêverie neuve», replaçant Camille au centre de son destin, dans la puissance et l'énergie de son caractère dont Claudel n'avait que trop bien compris les dangers et les failles. 